\title{
Land Cover and Habitat Classification from Earth Observation Data: A New Approach from BIO_SOS
}

\author{
Richard LUCAS ${ }^{1}$, Gwawr JONES ${ }^{1}$, Peter BUNTING ${ }^{1}$, Vasiliki KOSMIDOU ${ }^{2}$, \\ Zisiz PETROU ${ }^{2}$, Jordi INGLADA ${ }^{3}$, Maria ADAMO ${ }^{4}$, Palma BLONDA ${ }^{4}$, \\ Sander MUCHER ${ }^{5}$ and Damien ARVOR ${ }^{6}$ \\ ${ }^{1}$ Aberystwyth University/UK ·rml@aber.ac.uk \\ ${ }^{2} \mathrm{CERTH}$, Thessaloniki/Greece \\ ${ }^{3}$ CESBIO, Toulouse/France \\ ${ }^{4} \mathrm{CNR}$, Bari/Italy \\ ${ }^{5}$ ALTERRA, Wageningen/Netherlands \\ ${ }^{6}$ IRD, Montpellier/France
}

This contribution was double-blind reviewed as extended abstract.

\begin{abstract}
As part of the Biodiversity Multi-Source Monitoring System (BIO_SOS), a new approach to the classification of Food and Agricultural Organisation (FAO) Land Cover Classification System (LCCS) classes from very high resolution (VHR) remote sensing data has been developed. These classes are also translated to General Habitat Categories (GHCs). Examples of the classification are presented for Cors Fochno in Wales but can be generated for any site where appropriate remote sensing data have been acquired. The system has been developed for operational monitoring of protected areas and their surrounds.
\end{abstract}

\section{Introduction}

Research undertaken as part of the EU-funded BIO_SOS project has led to the development of the Earth Observation Data for Habitat Monitoring (EODHAM) system for consistent mapping of land cover (LC) classes and their subsequent translation to categories of habitat appropriate to support conservation agencies and land managers in decisions relating to protection of Natura 2000 sites in Europe. The primary input data sources are very high resolution $(<2 \mathrm{~m}$; VHR) EO-based measurements and on-site data, including ancillary information and in-field measurements. For this purpose, the Food and Agricultural Organisation (FAO) Land Cover Classification Scheme (LCCS) and the General Habitat Categories (GHCs), from which Annex I Habitats can be defined, have been proposed for describing LC and habitat categories. Key criteria in the design of the system include a) ease of use by end users (e.g., land managers, conservation agencies) and b) use with a defined range of satellite and, in some cases, airborne (e.g., LiDAR) data. 


\section{Basis of the EODHAM System}

The FAO-LCCS hierarchical classification scheme provides a logical framework for the integration of multi-source data, including from airborne and spaceborne imagery. The LCCS classification has been designed to provide standardised classes that can be applied and are recognised internationally. Key benefits are descriptions of a wide range of land covers, including those associated with agriculture. The scheme also considers environmental attributes (e.g., altitude, climate) as well as other technical attributes (e.g., crop-type). The classification is also scale independent.

As input to the classification, remote sensing data from a range of sensors can be integrated including optical and radar. In most cases, at least two dates of EO data (particularly in seasonal climates) are needed to characterise land surfaces, and particularly vegetation with marked phenologies. The pre-processing is crucial and involves radiometric and correction to Top of Atmosphere (TOA) reflectance, with capability for surface reflectance retrieval and topographic correction. Two primary layers representing 'large' and 'small' objects are generated from multi-temporal imagery, with these ideally acquired during periods of peak flush and senescence (i.e., winter/summer or wet/dry). Each layer object is then associated with a raster attribute table, the columns of which are populated with spectral and derived data (e.g., vegetation indices, object length/width ratio, area etc.) with these then used in the subsequent classifications. Within the large objects, broad features of the landscape are classified, including agricultural and forestry units of varying size and both urban and water features. Within the small object layer, a range of independent segmentations is implemented to specifically detect shape features including those that are linear (e.g., ditches, hedgerows, roads), rectangular (e.g., permanent and temporary dwellings) or circular/elliptical (e.g., individual tree crowns), with these classified using spectral and contextual rules. Remaining objects in the landscape are then generated using a generic segmentation based only on spectral information. Additional ancillary information can also be used, where appropriate, including digital elevation models, infrastructure and cadastral information. Information contained within the large and small object layers is then combined to describe more complex landscape components (e.g., olive groves, caravan parks, field units).

A rule-based classification is then applied and involves the creation of a series of layers (Tab. 1) with these representing the stages in the hierarchical but dichotomous decisions used in the FAO LCCS scheme. Spectral reflectance data but also a range of indices that quantitatively represent the relative amounts of, for example, photosynthetic, non-photosynthetic and aquatic vegetation as well as moisture content, surface roughness and vegetation structure.

The system benefits from the inclusion of height information such as that provided by LiDAR as this facilitates improved discrimination of lifeform and some non-form categories. Context sensitive features can be integrated in the classification of each layer, with these including shape and geometry indices as well as neighbourhood measures.

The classification focuses specifically on mapping various land cover characteristics associated with lifeform (e.g., cover, height, leaf type, phenology) and non-lifeform (e.g., urban density, water dynamics, surface aspect) categories, as defined by the LCCS. The LCCS codes (e.g., A1 for woody, A2 for herbaceous) generated for each layer are then combined to identify the final LCCS category at both Levels 1-3 and 4. Once LCCS 
categories have been mapped, these are translated through look up tables to GHCs from which key information (e.g., Annex I Habitats, detailed species maps) can be generated. Automated tools for accuracy assessment are provided.

Table 1: Main attribute columns populated for subsequent classification to LCCS Level 3 and beyond

\begin{tabular}{llll}
\hline Vegetation & Physical status & Bare Areas & Cultivation \\
\hline Lifeform & Water state & Surface aspect & Crop lifeform \\
Cover & Dynamics & Materials & Field size \\
Height & Persistence & Macropattern & Water seasonality \\
Leaf type & Duration & & Cultural \\
Phenology & Tidal/not tidal & & Crop combinations \\
Stratification & Substrate material & & \\
Distribution & Depth & & \\
Floating/rooted & Sediment loads & & \\
\hline
\end{tabular}

${ }^{1}$ Water, ice or snow

The LCCS scheme provides the opportunity to generate a very detailed classification of the landscape, which can compromise the interpretability of the map. This is particularly the case for woody vegetation where a large number of height and cover combinations result in a proliferation of the LCCS codes. For this reason, a generalized colour scheme has been developed which is universal such that new legends do not need to be generated when new LCCS combinations are generated for a site. The colour scheme has been designed based on the attributes of the LCCS. In particular, each LCCS qualifier in the individual layers corresponds to an attribute of colouring that is applied sequentially and reflects the appearance of the land cover in the landscape. A similar colour scheme has been developed also for GHC classes.

\section{Case study: Cors Fochno, Mid Wales, UK}

The Cors Fochno site in Wales was classified using the EODHAM system and a combination of Worldview-2 surface reflectance data that captured the 'stable' pre and peak flush periods (March 2012 and July 2011 respectively) as well as the post flush transition period (November, 2011). In addition, LiDAR data were also available, which were used primarily to quantify vegetation height.

For the site, key features in the landscape that required identification within the small object layer included buildings and temporary structures (e.g., caravans) and hedgerows. For these, specific tools for feature extraction outlined in the previous section were used, with the remaining landscape segmented using broad segmentation procedures. Larger objects were associated with fields comprised primarily of improved grasslands and managed forestry plantations, with the latter associated with land units that were largely occupied by woody vegetation (in the small object layers). 
The classification of LCCS categories is provided in Fig. 1. A large of classes (over 120) has been generated, partly because of the inclusion of height and cover categories generated from the LIDAR and Worldview-2 data. Hence, the legend is not included here. The classification however reflected the distribution of land covers observed within the area, including the active bog, perennial closed grasslands (towards margins), different forest types and urban areas. The accuracy in the classification of the majority of LCCS classes exceeded $70 \%$, but further validation is being undertaken with reference to aerial photography, unmanned airborne system (UAS) and field data to be collected in 2013.

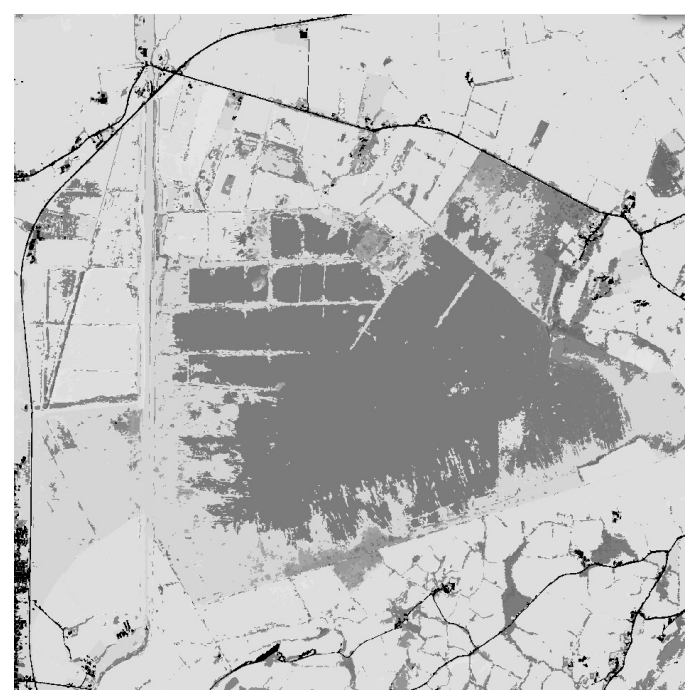

Fig. 1:

Classification of LCCS categories, Cors Fochno and surrounds. Over 120 classes are defined with these associated with broadleaved (dark green) and mixed forests (brown) of varying height and cover, perennial grasslands (mid green), agricultural fields (yellow), active raised bog (purple), urban areas (black) and open water (grey).

\section{Conclusions}

The research within BIO_SOS has led to the development of the EODHAM system as a comprehensive open-source and consistent tool for land cover and habitat mapping from VHR but also coarser spatial resolution imagery, which can be applied anywhere worldwide given appropriate data. The system benefits from the inclusion of LiDAR data, which facilitates improved discrimination of lifeform and some non-form categories. Height information of the vegetation is crucial in the discrimination of the LCCS and GHC categories and can be directly derived from LiDAR data although, if necessary, indirectly from spectral information in the VHR optical imagery. Future work is focusing on refining components of the system (and further operationalization for end-users) and integrating a change detection module. The system is being applied to a range of Natura 2000 sites throughout Europe and its use at sites (e.g., in India and Brazil) is being evaluated before the conclusion of the BIO_SOS project. 